\title{
APPLIED STRUCTURAL DETAIL ENHANCEMENT METHODS IN VIDEOENDOSCOPIC IMAGING AND THEIR USE IN CLINICAL PRACTICE
}

\author{
Katsiaryna Halavataya ${ }^{1^{*}}$, Tatyana Ginko², Ludmila Kalatskaya ${ }^{1}$ \\ ${ }^{1}$ Belarusian State University, Minsk, Belarus \\ ${ }^{2}$ Minsk City Clinical Oncologic Dispensary, Minsk, Belarus
}

\begin{abstract}
Introduction of laser systems to clinical practice significantly amplifies the abilities of endoscopic patient treatment. The software used in endoscopic systems does not always allow us to take into account the specific features of image processing for analyzing cancer tumors. The article provides an overview of structural detail enhancement methods in videoendoscopic imaging and implementation of these methods developed using ImageJ plugin API.
\end{abstract}

Key words: Videoendoscopic systems, photodynamic therapy, fluorescent component, color images

DOI: $10.21175 / \operatorname{RadProc} .2016 .33$

\section{INTRODUCTION}

There is a very broad array of image processing methods aimed at enhancing their visual qualities, accentuating some of their features and even detecting shapes and objects. Thus, developing effective and accurate methods for programmatic processing and analysis of images significantly simplifies diagnostics.

An important factor in endoscopic image processing is the effectiveness of used processing methods. Quite often, one of the requirements for such processing is that it must occur in real-time as to be applied to live image feed from the endoscope, so most of the methods discussed here are optimized for effective operation over streamed image context, like video.

The paper also researches images acquired after using photodynamic therapy (PDT). Photodynamic therapy is one of the evolving methods of diagnosis and treatment of oncological pathologies. PDT is based on the usage of special drugs called photosensitizers solutions that are sensitive to visible light, and lowintensity laser emission with a wavelength that corresponds to the peak absorption of a photosensitizer. Despite the fact that the research of PDT has been conducted for many years, it was not used in clinical practice until recently, mainly because there were little to none non-toxic photoactive drugs to be used in this method [1].

To increase the quality of photodynamic diagnosis and analysis of treatment efficiency, a proper computer-aided image processing for PDT images is required. The functionality of such a diagnostic may vary, but the defining factors are: pre-processing (increasing image quality, noise filtering, sharpness enhancement), detail enhancement for researched areas, such as the areas where the photosensitizer is concentrated, producing necessary statistical information (e.g. percentage of photosensitizer concentration in the tissue) and analyzing how the acquired data diverges from the expected or normal state.

The aim of this paper is the analysis and development of glare detection, size calculation and fluorescent component detection, taking into account the specific features of image processing for endoscopic systems in real time.

To review, in detail, the process and algorithms of color endoscopic image analysis, all researched methods are implemented as a standalone software package based on ImageJ image processing platform plug-in system, using Java programming language. The platform provides a simple and focused object-oriented API with various built-in functions for image processing, which greatly simplifies the process of implementing researched algorithms. Moreover, it is possible to use Java language toolkit to create a crossplatform graphical user interface that does not require the user to know the details of used methods and algorithms.

\section{DEVELOPED METHODS}

\subsection{Glare detection in videoendoscopic imaging}

Glares appear on videoendoscopic images because, as part of endoscopic research, endoscopes themselves are equipped with a powerful light source, while organism internal organs are covered by a layer of mucus. Glares may negatively affect the accuracy of

\footnotetext{
*katerina-golovataya@yandex.ru
} 
research and result in incorrect automated analysis based on the brightness of the image.

There are two primary approaches to removing negative glare influence: completely removing glares from all analysis and statistical research, and replacing glare areas with homogeneous (in respect to surrounding areas) filling. Both of these approaches require detecting glare areas on the image.

Detecting glares can be performed using pointrecursive filling algorithm. This algorithm allows the detection of relatively homogeneous areas of the image, i.e. areas where color doesn't change, allowing us to effectively select areas with sharp edges, such as glares.

The algorithm itself comprises several steps. The first thing that is required is finding centerpoints points which are guaranteed to be inside the glare area. The simplest and the most effective approach here is to choose the local maximal points of the image. The problem here is that impulse (high-frequency) noises that are presented in the image may produce additional local maxima, thus, they must first be removed by smoothing the image. Smoothing can be performed by convoluting the image with Gaussian blur operator. So the first step of the algorithm is smoothing.

The second step is finding local maxima. The simplest way to do so is to detect points that have higher brightness than 4 surrounding points (top, bottom, left and right). 4-point traversal can potentially exclude maxima where it is local over diagonal surrounding points. This can be easily fixed by replacing it with 8-point traversal, where diagonal points (lower left, lower right, higher left and higher right) are used with correcting weight factors. Also, when working with glares on the image, it is important to note that only high-brightness local maxima are relevant. There may be lots of local maxima because of the surface unevenness in the image, so it's important to ignore these and only include maxima with a brightness value above a certain threshold, which can be determined interactively or experimentally [2].

The third step is the actual point-recursive fill. It is possible to use both 4-point and 8-point traversal. This step also requires a certain brightness difference parameter, which can be determined interactively or experimentally. The algorithm is the following: surrounding points are traversed, and for each point its brightness value is compared to the current point. If the brightness difference is higher than the brightness difference parameter, the point is ignored. Otherwise, it is included in the area and its neighbours are also traversed. It is important to keep track of already included points in order not to traverse the same points indefinitely [3].

Point-recursive fill algorithm allows the detection of relatively homogeneous areas of the image. Thus, it is perfect for detecting glares in order to exclude them from processing. The result of point-recursive glare detection on a sample image is shown in Figure 1.

The implementation of point-recursive fill in developed software complex is interactive, i.e. it is possible to adjust threshold values (and immediately see the result) until the quality of glare detection is practically sufficient.

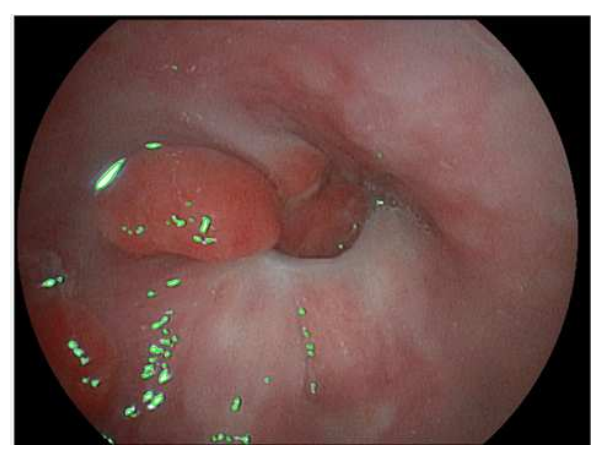

Figure 1. Result of glare detection. Detected glares are outlined in green

\subsection{Determining the size of objects on color images}

One of the most important parameters in diagnostics is the size of the researched tumor area, as well as its growth or shrinking before, during and after treatment. The size of the tumor area can be used as a measure of effectiveness of used treatment.

It is possible to measure an absolute and relative area of the tumor surface. Measuring the absolute value of the area is quite hard, because it requires not only the use of the information from the image, but also knowledge of specific optical details (zoom, focus distance, etc.) about the hardware used, as well as about the distance to the particular researched point in the image. Relative area can be measured based on different parameters, but it is important for it to be informative - i.e. there should be a possibility to use it in comparison with previous and future researches.

The proposed method for measuring the relative area is based on selecting an ellipse based on the projection of esophagus clearance, followed by calculating the part of the area of interest that overlaps with the projection ellipse (Figure 2).

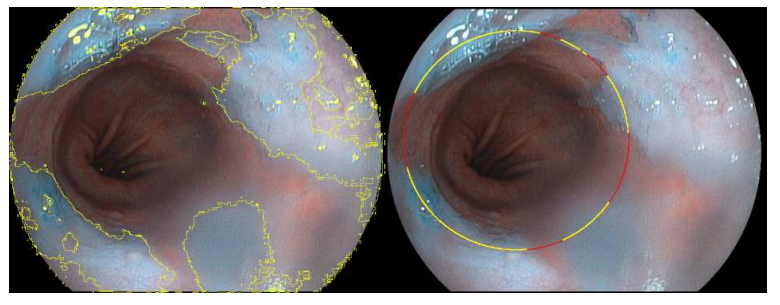

a)

b)

Figure 2. Determination of the relative size of the affected part:

a) Selected affected area, outlined in yellow; b) Affected area overlap (yellow) with projection ellipse (red)

Determining the absolute area is based on including the objects of known size in the image. One of the most convenient objects in this sense is the endoscope tube. Its size is always determined and is known to the specialist conducting the research. Figure 2.6 provides the use of such method.

Thus, image processing systems enable us to greatly enhance visual image quality, as well as detect regions of interest on the image. This chapter went over algorithms of structural detail enhancement and image processing that can be used for images acquired from videoendoscopy and treatment endoscopy systems. 
Point-recursive fill algorithm was also shown to be incredibly effective at determining glares on the image.

Fluorescent component selection can be used to provide visual clues about the researched area. Determination of absolute or relative area of a pathological surface can greatly aid in stating the diagnosis and in analyzing the progression of disease and treatment.

It is important to note that relative size calculation is only informative in comparisons, i.e. it can be used as a measure of disease progression over a course of multiple endoscopic researches. Usually, for concrete clinical applications, absolute size calculation is required. However, calculating absolute size, as noted above, can only be correctly performed if there is a known-size object present on the image. The error of absolute size calculation using this method is around $10 \%$ when compared to traditional methods (e.g. endosonography), which is usually acceptable for making strategic treatment decisions.

\subsection{Selecting fluorescent component}

Photodynamic therapy (PDT) is based on the use of special drugs called photosensitizers - substances that are sensitive to light and to low-intensity laser emission with a wavelength corresponding to the absorption peak of a photosensitizer. In PDT, the photosensitizer is administered to patient intravenously, after which photosensitizer accumulates in tumor cells. After that, the affected area is irradiated with a low-intensity laser emission. The wavelength of the radiation is determined by the absorption spectrum of the sensitizer and corresponds, as mentioned above, to the maximum absorption of the sensitizer used.

Detecting fluorescent components on PDT images is based on converting input colorspace to another algebraic space, where image points are spread mostly along the basis vectors. This method is mainly possible because of the specific features of PDT images - they have a very distinct point distribution across the colorspace (Figure 3, Figure 4a).

As shown in Figure 1, most of the image points are distributed across one plane. The color changes from black to light blue on maximum point spread vector, which corresponds to brightness distribution in the image. Perpendicular vector, along which the color changes from pale blue to cyan, corresponds to the fluorescence of the accumulated photosensitizer. Along the third perpendicular vector, which is directed as a normal to the points plain, the points spread is very low. This spread is mostly present because of noises in the image.

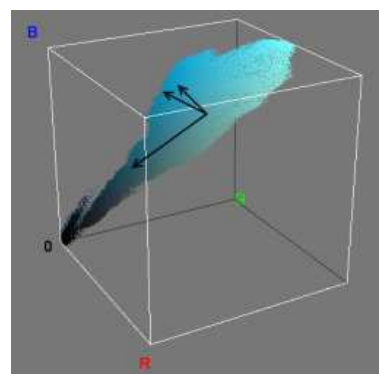

Figure 3. RGB colorspace of a PDT image on Figure 4a)
Fluorescent component makes fluorescence visible and distinct and can be used for structural detail enhancement of objects on videoendoscopic images, e.g. non-linear contrast enhancement [4]. An example of using PCA for non-linear contrast enhancement is shown on Figure 4.

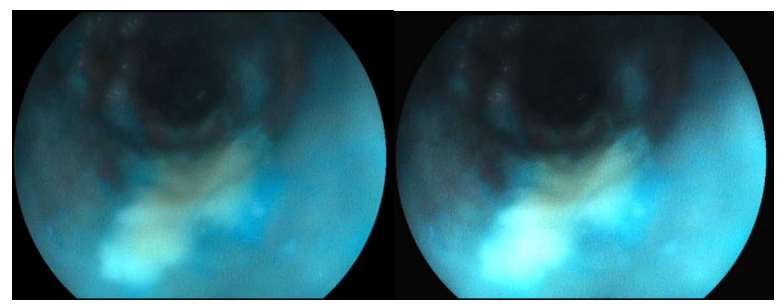

Figure 4. Non-linear PCA filtering

a) Source image b) Enhanced image

\section{IMPLEMENTATION}

Videoendoscopic expert systems are an important part of medical research, as they allow us to identify the disease at any stage of its progression and take appropriate treatment measures in timely fashion. To ensure high quality analysis of the images, both structural detail enhancement and pre-processing are required.

The emergence of new methodologies in medical diagnostics and therapy demands creating new mechanisms of image processing that take into account the nuances of specific methodology. This includes special processing for photodynamic therapy images, building $3 \mathrm{D}$ model of the researched area for volumetric irradiation distribution planning.

The goal of the developed software complex is to provide specialized algorithms for pre-processing, structural detail enhancement, noise filtering and statistical analysis of medical images.

The project is a complex of various software packages to simplify diagnosis and increase effectiveness of conducted endoscopic research. It is based on the implementation of various algorithms of pre-processing and structural detail enhancement of color images acquired using videoendoscopic systems. Implemented algorithms for pre-processing include contrast enhancement, sharpness enhancement and noise filtering. Structural detail enhancement contains multiple steps, including tumor detection and surface structure detail enhancement. Moreover, it also includes algorithms for working with photodynamic therapy imaging and narrow-band imaging.

Application user interface is based on Swing desktop GUI library for Java language platform. User interface also provides a way to tweak some of the algorithms interactively, i.e. displaying the result of the processing as the parameters for processing change.

The system is designed on a platform of image processing software ImageJ [5] and implemented using Java programming language. The complex is developed as a cross-platform implementation and works with the majority of devices and platforms that support Java. For the implementation of the user interface, Swing library is used. Matrix algebra operations are implemented using JML library. 
User interface of the developed software complex is shown on Figure 5.

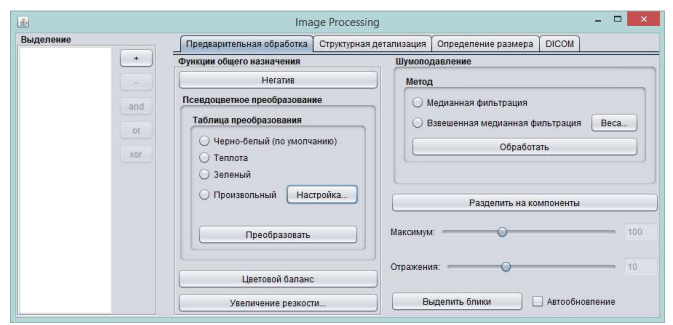

Figure 5. User interface of the developed software complex, pre-processing tab

\section{CONCLUSION}

As shown in the research paper, videoendoscopic image processing simplifies diagnosis and can be used to automate the process of clinical endoscopic research. It is also important that image processing methods and algorithms take imaging specifics into account, e.g. PDT-specific image processing.

An implemented software complex allows the use of various methods and algorithms discussed in this paper in clinical research.
The uniqueness of the developed imaging system is that it contains the implementation of the specialized image processing techniques developed for specific applications. The development model is built in such a way that, if necessary, it is possible to enhance the functionality of the system by providing additional plugins.

\section{REFERENCES}

1. Advances in Photodynamic Therapy: Basic, Translational and Clinical, M.R. Hamblin and P. Mroz, Eds., Norwood (MA), USA: Artech House, 2008

2. T. Ginko, K. Halavataya, L. Kalatskaya, "Processing and Structural Detail Enhancement of Imaging Objects in Treatment Endoscopy," Electron. Inf. (Электроника инфо), vol. 2, pp. 27-31, 2014

3. R. Gonzales, R. Woods, Digital Image Processing, 3rd ed., Upper Saddle River, (NJ), USA: Prentice Hall, 2007

4. T. Stehle, Image Processing for Endoscopy: Algorithms and Real-Time Implementations, Göttingen, Germany: Sierke, 2011

5. Website of ImageJ:

Retrieved from: http://rsbweb.nih.gov/ij/index.html Retrieved on: 09.12.2015 